\title{
On Some Aspects of the Dynamics of Dispersed Water in the Forest in the Presence of a Fire
}

\author{
M. N. Ilicheva \\ Nizhny Novgorod State Technical University named after R.E. Alekseeva (NNSTU), \\ Nizhny Novgorod, 603950, Russia \\ poman99@mail.ru
}

\begin{abstract}
This paper presents the results of modeling the droplets of different sizes adhesion to vegetation elements. The paper deals with issues related to extinguishing a forest fire using dispersed water. A key factor in the effectiveness of fire extinguishing is to ensure the delivery of water to the fire site and the absorption of energy due to the vaporization process. It is assumed that droplets do not affect the velocity field of the gas phase. Their movement is due to inertia and the effect of the force of aerodynamic drag. It is shown that the logit of the fraction of adhered droplets is close to linear with the logarithm of the size, which makes it possible to estimate the permeability of the forest for dispersed water particles. It is shown in the work that smaller droplets are better flow around obstacles.
\end{abstract}

Keywords: numerical modeling, dispersed water, aerodynamics, gas-dispersed medium, forest fire extinguishing

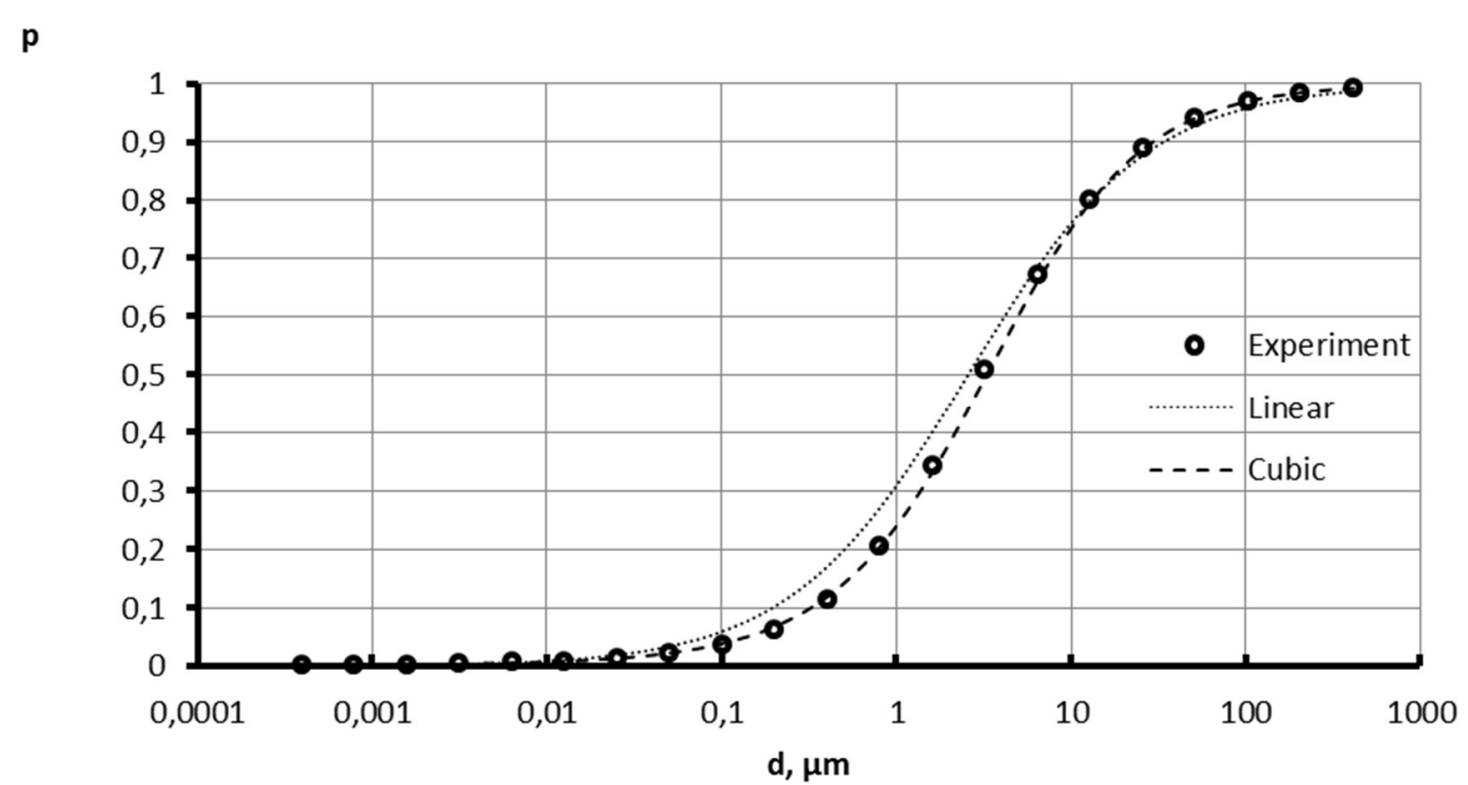

Linear and cubic approximation of numeric experiment of the particle adhering probability 


\title{
О некоторых аспектах динамики дисперсной воды в лесном массиве при наличии пожара
}

\author{
М. Н. Ильичева \\ Нижегородский государственный технический университет им. Р. Е. Алексеева, \\ Россия, г. Нижний Новгород, 603950, ул. Минина, 24 \\ poman99@mail.ru
}

\begin{abstract}
Аннотация
В данной статье приводятся результаты моделирования налипания капель разного размера на элементы растительности. В работе рассматриваются вопросы, связанные с тушением лесного пожара при помощи дисперсной воды. Ключевым фактором эффективности пожаротушения является обеспечение доставки воды в очаг пожара и поглощение энергии за счёт процесса парообразования. Предполагается, что капли не влияют на поле скоростей газовой фазы. Их движение обусловлено инерцией и воздействием силы аэродинамического сопротивления. Показано, что логит доли налипших капель находится в зависимости близкой к линейной от логарифма их размера, что позволяет оценить проницаемость лесного массива для дисперсных частиц воды. В работе показано, что более мелкие капли лучше обтекают препятствия.
\end{abstract}

Ключевые слова: численное моделирование, дисперсная вода, аэродинамика, газодисперсная среда, тушение лесного пожара

\section{1. Введение}

Лесные пожары остаются основной причиной гибели лесов и в Российской Федерации и за ее пределами.Использование авиационных технологий в удаленных и труднодоступных местах часто становится единственным способом ликвидации горения. Экспериментальные и теоретические исследования показали, что простой сброс большого количества воды не эффективно тушит пожар[1, 2]. Большая часть сброшенной воды уносится конвективной колонкой, часть водыпод воздействием высокой температуры испаряется.Математическое моделирование подтверждает эти факты [3]. Динамика тушения пожара существенно зависит от способа и точности подачи воды в зону горения [4-6]. Так, в работе [4] авторы рассматривают упрощенную модель тушения лесного пожара на основе сброса воды. В работе предполагается, что вся вода пролетает сверху вниз, а при попадании в нагретую среду происходит испарение до исчерпания воды или снижения температуры среды до точки кипения воды. В работе [5] рассматривается мгновенная подача воды в нужную точку без потерь с учетом её нормального пространственного распределения. Тушение лесного пожара при подаче воды сверху на основе псевдо-трехмерной постановки рассмотрено в [6]. В работах [4-6] не рассматриваются потери воды, связанные с её уносом и налипанием на элементы растительности.

Авторами работы [7] получены точные экспериментальные данные по свободному падению с высоты объемов воды в различных условиях с использованием современных средств видеорегистрации и визуализации. В результате показано, что при свободном падении ядра воды объемом $0.1 \div 0.5$ л с высоты 5 м формируется облако мелкодисперсных частиц, которое под воздействием аэродинамических сил растет в вертикальном и горизонтальном направлении [7]. Дисперсная вода не только оседает и испаряется, но также может стекать, слипаться, разрушаться и сдуваться ветром. 
Моделирование полного комплекса процессов, динамики полидисперсной фазы воды при тушении лесного пожара является весьма сложной отдельной задачей. Представляют большой интерес процессы динамики дисперсной фазы в лесной растительности при наличии пожара. Ключевыми процессами, влияющими на динамику самого пожара при подаче дисперсной воды, является её способность налипать на растительность и поглощать тепло при испарении. В представленной работе рассматривается задачамоделирования динамики дисперсных частиц воды конечного размера при движении в слое растительности. Задача состоит в определении характеристик движения дисперсной воды в слое растительности, и её оседание на элементы лесных горючих материалов. Очень мелкие частицы облетают элементы легкогорючих материалов вместе с потоком воздуха, не сталкиваясь с ними.

\section{2. Вероятностная модель налипания мелкодисперсных частиц на недеформируемую преграду}

Рассматривается двумерная задача моделирования налипания дисперсных частиц на тело в форме прямоугольника, обтекаемое газодисперсным потоком. В начальный момент частицы находятся перед телом на расстоянии, при котором поле скоростей можно считать невозмущенным. Затем частицы движутся вместе с газовым потоком под воздействием силы лобового сопротивления.

Рассмотрим систему уравнений в виде

$$
\begin{gathered}
\frac{\partial \rho_{5}}{\partial t}+\frac{\partial\left(\rho_{5} U\right)}{\partial x}+\frac{\partial\left(\rho_{5} W\right)}{\partial z}=0 \\
\frac{\partial\left(\rho_{5} U\right)}{\partial t}+\frac{\partial\left(\rho_{5} U^{2}\right)}{\partial x}+\frac{\partial\left(\rho_{5} U W\right)}{\partial z}=\frac{\partial P}{\partial x}+\frac{\partial}{\partial x}\left(\mu_{t} \frac{\partial U}{\partial x}\right)+\frac{\partial}{\partial z}\left(\mu_{t} \frac{\partial U}{\partial z}\right) \\
\frac{\partial\left(\rho_{5} W\right)}{\partial t}+\frac{\partial\left(\rho_{5} U W\right)}{\partial x}+\frac{\partial\left(\rho_{5} W^{2}\right)}{\partial z}=\frac{\partial P}{\partial x}+\frac{\partial}{\partial x}\left(\mu_{t} \frac{\partial W}{\partial x}\right)+\frac{\partial}{\partial z}\left(\mu_{t} \frac{\partial W}{\partial z}\right), \\
P=\frac{\rho_{5} R T}{M}
\end{gathered}
$$

Граничные условия имеют следующий вид:

$$
\begin{aligned}
& z=h_{\max }: U=U_{e}, W=\frac{P-P_{e}}{P} \sqrt{\frac{R T}{M}}, \quad z=0: \mu_{t}=0, U=U_{e}, W=0 ; \\
& x=0: \quad U=U_{e}-\frac{P-P_{e}}{P} \sqrt{\frac{R T}{M}}, W=0 ; \\
& x=x_{\max }: U=U_{e}+\frac{P-P_{e}}{P} \sqrt{\frac{R T}{M}}, W=0 ; \\
& G: \quad \mu_{t}=0, U=0, W=0
\end{aligned}
$$

На первом этапе моделирования решается стационарная задача обтекания (1)-(8) для определения поля скоростей при обтекании элемента растительности.

На втором этапе на основе уравнений (9)-(10) выполняется моделирование движения частиц с различным значением коэффициента аэродинамического сопротивления. В начальный момент, частица имеет координаты $x_{p, 0}, z_{p, 0}$ и движется со скоростью среды в этой точке. Движение частицы происходит под действием аэродинамического сопротивления за счёт силы тяжести и инерции. 


$$
\left\{\begin{array}{l}
\frac{\mathrm{d} x_{p}}{\mathrm{~d} t}=U_{p}, \\
\frac{\mathrm{d} z_{p}}{\mathrm{~d} t}=W_{p}, \\
\frac{\mathrm{d} U_{p}}{\mathrm{~d} t}=-c_{d}\left(U_{p}-U\right) \sqrt{\left(U_{p}-U\right)^{2}+\left(W_{p}-W\right)^{2}}, \\
\frac{\mathrm{d} W_{p}}{\mathrm{~d} t}=-c_{d}\left(W_{p}-W\right) \sqrt{\left(U_{p}-U\right)^{2}+\left(W_{p}-W\right)^{2}}-g
\end{array}\right.
$$

Начальные условия для капли имеют вид

$$
x_{p}(0)=x_{p, 0}, z_{p}(0)=z_{p, 0}, U_{p}(0)=U\left(x_{p, 0}, z_{p, 0}\right), W_{p}(0)=W\left(x_{p, 0}, z_{p, 0}\right),
$$

где $x, z$ - горизонтальная и вертикальная координата, м; $U, W$ - составляющие скорости газовой фазы по направлениям $x$ и $z$ соответственно, м/с; $U_{e}-$ скорость невозмущенного потока газовой фазы относительно обтекаемого тела; $\rho_{5}-$ плотность газовой фазы, кг $/ \mathrm{m}^{3} ; P$ - давление, Па; $P_{e}$ - давление невозмущённой среды на нулевой высоте; $\rho_{e}-$ плотность невозмущённой среды на нулевой высоте; $\mu_{t}-$ коэффициент динамической вязкости воздуха, Па·с; $R-$ универсальная газовая постоянная, $\mathrm{M}^{2} \cdot \mathrm{K \Gamma}^{\cdot} \mathrm{c}^{-2} \cdot \mathrm{K}^{-1} \cdot \mathrm{Moль}^{-1} ; T=300 \mathrm{~K}$ - температура окружающей среды; $M$ - молярная масса воздуха, кг/моль; $x_{\max }, h_{\max }$ - длина и высота расчётной области соответственно, м; $x_{p}, z_{p}$ - координата мелкодисперсной частицы, м; $U_{p}, W_{p}$ - составляющие скорости мелкодисперсной частицы по направлениям $x$ и $z$ соответственно, м/с; $c_{d}-$ коэффициент аэродинамического сопротивления мелкодисперсной частицы, $\mathrm{M}^{-1} ; c_{x}-$ коэффициент лобового сопротивления мелкодисперсной частицы; $\Delta V$ - модуль разности между скоростью движения мелкодисперсной частицы и воздуха вокруг неё, м/c; $m$ - масса мелкодис-

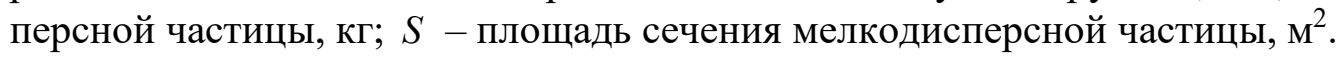

При попадании в ячейку, принадлежащую обтекаемому телу частица прилипает к телу и для нее вычислительный процесс останавливается. При достижении частицей границ расчетной области расчеты также прекращаются.

Будем искать зависимость изменения диаметра частицы от коэффициента аэродинамического сопротивления. Величина его силы с учетом шарообразной формы капли, вычисляется по формуле

$$
F=m \frac{\mathrm{d} U_{p}}{\mathrm{~d} t}=m c_{d}(\Delta U)^{2}
$$

где $\Delta U, \mathrm{M} / \mathrm{c}$ - разность между скоростью частицы и газовой среды

После преобразований получим

$$
\begin{gathered}
F=m \frac{\mathrm{d} U_{p}}{\mathrm{~d} t}=m c_{d}(\Delta U)^{2}=c_{d}(\Delta U)^{2}\left(\frac{4}{3} \pi r^{3}\right) \rho_{p}, \\
F=\frac{c_{x} \rho_{e} \cdot S_{p r} \cdot \Delta U^{2}}{2}=\frac{c_{x} \rho_{e} \cdot \pi r^{2} \cdot \Delta U^{2}}{2}
\end{gathered}
$$

Находим соотношение, связывающее диаметр частицы и её коэффициент аэродинамического сопротивления

$$
c_{d}(\Delta V)^{2}\left(\frac{4}{3} \pi r^{3}\right) \rho_{p}=\frac{c_{x} \rho_{e} \cdot \pi r^{2} \cdot \Delta V^{2}}{2}
$$




$$
\begin{gathered}
c_{d}\left(\frac{4}{3} r\right) \rho_{p}=\frac{c_{x} \rho_{e}}{2}, \\
c_{d}=\frac{3 c_{x} \rho_{e}}{8 r \rho_{p}}=\frac{3 c_{x} \rho_{e}}{4 d \rho_{p}}, \\
d=\frac{3 c_{x} \rho_{e}}{4 \rho_{p} \mathrm{~A}_{\mathrm{coeff}}},
\end{gathered}
$$

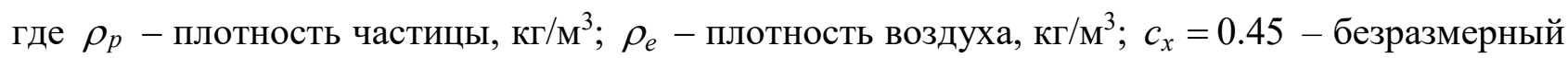
коэффициент лобового сопротивления сферы

Будем считать, что при $c_{d}=10^{18}$ дисперсные частицы не должны налипать на тело, так как движутся вместе с потоком газа, который не проникает в элемент растительности. Следовательно, частицы, которые налипают при $c_{d}=10^{18}$ исключается при вычислении доли прилипших частиц из числа тех, которые могли не прилипнуть. Далее выполняется аппроксимация логита доли налипших частиц методом наименьших квадратов в форме

$$
L f r\left(L U_{e}, L c_{d}\right)=a_{1}+a_{2} L U_{e}+a_{3} L c_{d}+a_{4} L c_{d}^{2}+a_{5} L c_{d}^{3},
$$

где $L U_{e}=\ln \left(U_{e}\right) ; L c_{d}=\ln \left(c_{d}\right) ; a_{i}, i=1, \ldots, 5$ - полиномиальные коэффициенты.

Решение задачи о налипании капли выполняется в два этапа. На первом этапе выполняется моделирование обтекания препятствия воздушными потоками на основе системы обыкновенных уравнений в частных производных при помощи метода крупных частиц [12]. На втором этапе выполняется моделирование динамики частиц, движущихся под действием силы тяжести и аэродинамического сопротивления среды на основе решения системы обыкновенных дифференциальных уравнений методом Эйлера. В начальный момент частицы размещаются на заданном расстоянии от грани препятствия, обращенной к внешнему полю скоростей. Для определения скорости среды по координатам частицы используется билинейная интерполяция. Низкий порядок точности связан с тем, что при решении задачи обтекания используется метод первого порядка точности по пространству. При попадании частиц в области, принадлежащей обтекаемому препятствию, её скорость считается равной нулю, что соответствует прилипанию. После моделирования определяется доля частиц, прилипших к препятствию. При достаточно большом значении аэродинамического сопротивления частицы не имеют инерции и движутся вместе с газовой фазой. В этом случае налипание частиц на препятствие связано с численной погрешностью интерполяции скорости. Доля таких частиц составляет от $3 \%$ до $6 \%$ в зависимости от скорости газовой фазы на границах. Для компенсации погрешности такие частицы исключаются из рассмотрения, что обеспечивается хорошее согласование с полиномиальной зависимостью между логарифмом размера частиц и логитом вероятности их прилипания.

\section{1. Результаты моделирования}

На рис. 1 показан результат решения задачи (1)-(8). На удалении от элемента растительности его влияние на скорость потока несущественно. Формируется квазистационарное ламинарное течение. На рис. 2-3 представлена зависимость диаметра дисперсной частицы от вероятности и логита вероятности налипания ее на тело.

Результаты аппроксимации находятся в хорошем согласовании с результатами численных экспериментов на основе решения задачи аэродинамики(1)-(10). Полученные результаты показывают, что можно решить численную задачу для небольшого набора параметров и форм препятствия и использовать полиномиальную аппроксимацию логита доли налипающих частиц от логарифма их размера, что позволяет сделать оценки возможности проникновения частиц воды в слой лесной растительности. 


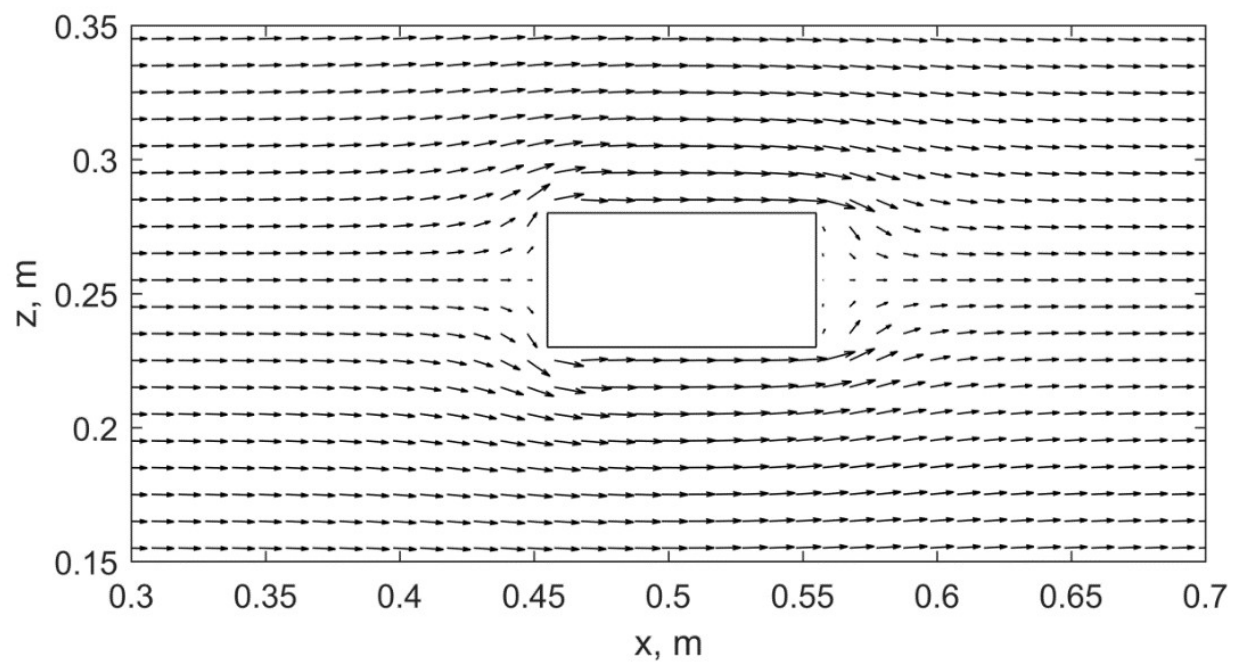

Рис. 1. Поле скоростей, сформированное при обтекании элемента растительности logit(p)

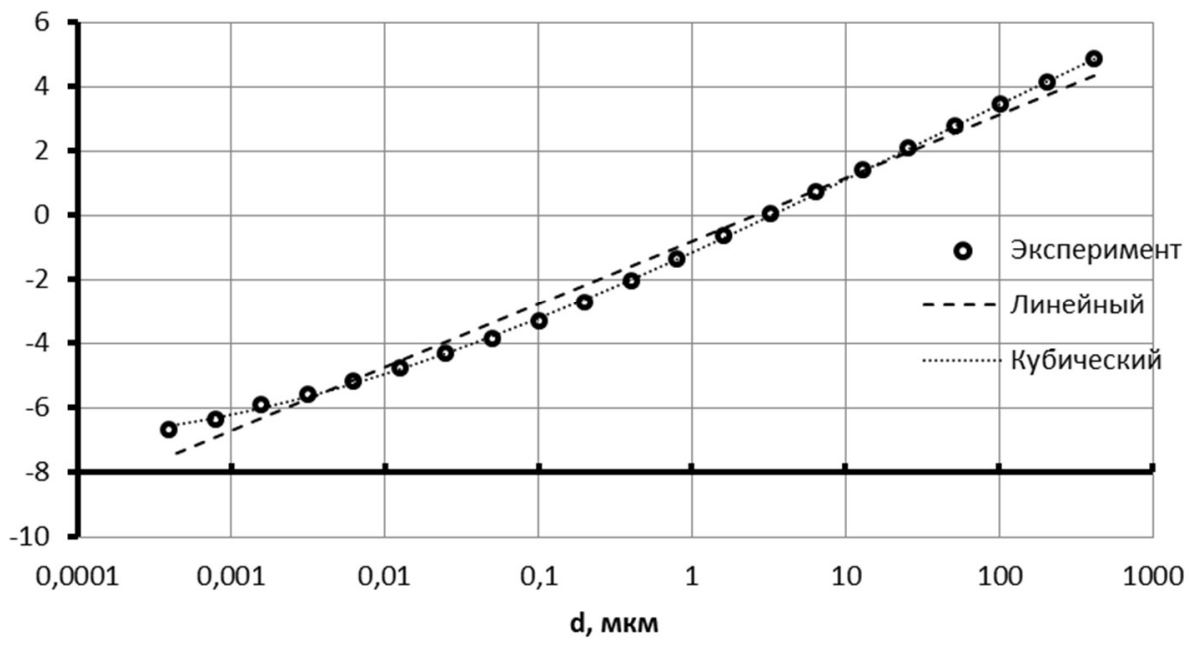

Рис. 2. Результат полиномиальной аппроксимации коэффициентов зависимости логита вероятности налипания от логарифма размера частиц

$\mathbf{p}$

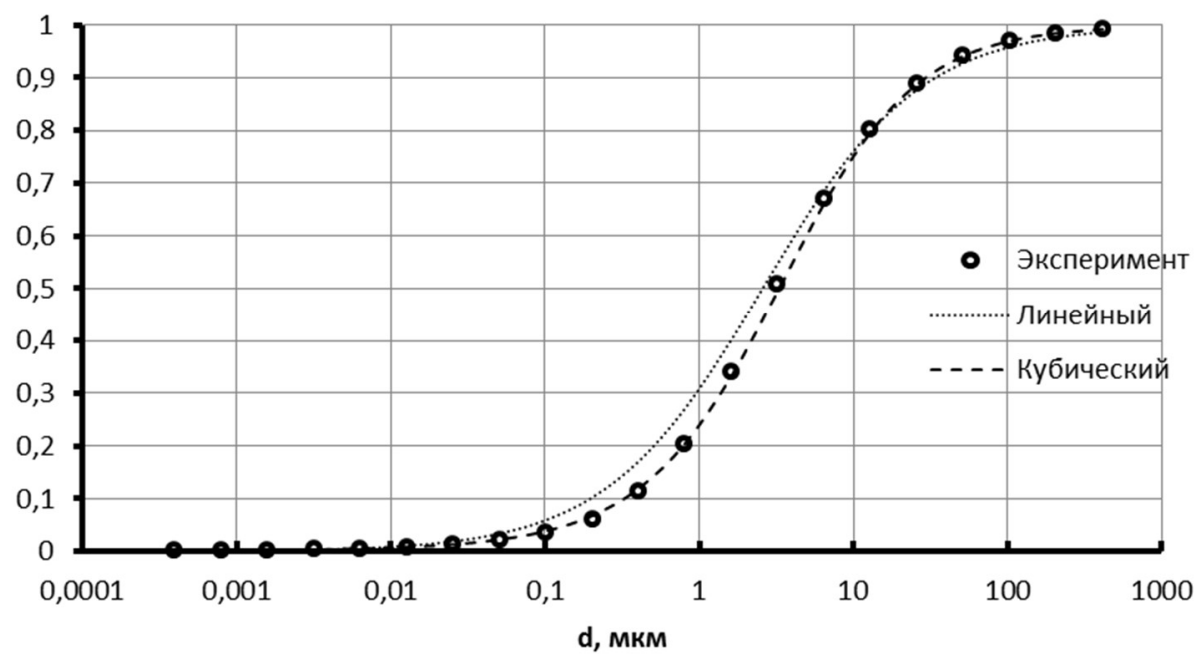

Рис. 3. Сравнение вероятности налипания, полученной на основе численного эксперимента и полиномиальной зависимости 


\section{3. Заключение}

Частицы большого размера не обладают способностью облетать элементы растительности вместе с газовым потоком. Для тушения пожара мелкими водными каплями целесообразно обеспечить их точную доставку в нужном количестве в зону уязвимости пожара. Одним из эффективных способов доставить воду в область горения является использование капсул в активной оболочке, которые позволяют преодолеть конвективную колонку. Этот способ смоделирован в работе $[13,14]$, где после разрыва капсулы, вода распыляется на частицы бесконечно малого размера, которые не налипают на растительность, и температура при этом мгновенно перераспределяется между каплями и средой.

\section{Литература}

1. Копылов Н.П., Карпов В.Н., Кузнецов А.Е., Федоткин Д.В., Хасанов И.Р., Сушкина Е.Ю. Особенности тушения лесных пожаровс применением авиации //Вестник Томского государственного университета. 2019. № 59. С. 79-86.

2. Глушков Д.О., Копылов Н.П., Кропотова С.С., Кузнецов Г.В., Стрижак П.А. Локализация и подавление горения лесных материалов с применением авиации. Новосибирск: Изд-во СО РАН, 2020. - 337 c.

3. Satoh K., Maeda I., Kuwahara K., Yang K. A Numerical Study of Water Dump in Aerial Fire Fighting // Fire Safety Science 8. 2005. Pp. 777-787. doi:10.3801/IAFSS.FSS.8-777

4. Лощилов С.А., Маслеников Д.А., Постнов А.Д., Катаева Л.Ю. Исследование влияния интенсивности сброса воды на динамику лесного пожара // Естественные и технические науки. 2013. № 6 (68). С. $37-40$.

5. Нищенков В.В., Романова Н.А., Катаева Л.Ю., Масленников Д.А., Лощилов А.А. Об оптимальной стратегии тушения лесного пожара водяной пушкой // Современные проблемы науки и образования. 2014. № 3. С. 692.

6. Kataeva L.Y., Maslennikov D.A., Loshchilova N.A. On the laws of combustion wave suppression by free water in a homogeneous porous layer of organic combustible materials // Fluid Dynamics. 2016. Vol. 51. No 3. Pp. 389-399.

7. Накоряков В.Е., Кузнецов Г.В., Стрижак П.А. О предельных поперечных размерах капельного облака при разрушении водяного массива в процессе падения с большой высоты // Доклады академии наук, 2017, том 475, № 2, с. 145-149. DOI: 10.7868/S0869565217020062

8. Flemmer R.L.C. and Banks, C.L., On the Drag Coefficient of a Sphere, Powder Technol., 1986, vol. 48, pp. 217-221.

9. Turton R. and Levenspiel O.A., A Short Note on the Drag Correlation for Spheres, Powder Technol., 1986, vol. 47, no. 1, pp. 83-86.

10. Катаева Л.Ю., Карпухин В.Б. О методе Гира численного моделирования динамических систем, описываемых жесткими обыкновенными дифференциальными уравнениями // Наука и техника транспорта, № 2. -М.:РГОТУПС, 2008.

11. Войтков И.С., Волков Р.С., Кузнецов Г.В., Стрижак П.А. Высокотемпературное испарение капель воды в газовой среде //Журнал технической физики, 2017, том 87, вып. 12. С.1911-1914.

12. Бабкин А.В. Численные методы в задачах физики быстропротекающих процессов: учебник для втузов. М.: Изд-во МГТУ им. Н.Э. Баумана, 2006. - 520 с.

13. Ильичева М.Н., Катаева Л.Ю. Математическое моделирование тушения лесного пожара капсулами с водой в разрушающейся оболочке // XXXII научно-практическая конференция Актуальные проблемы пожарной безопасности, ВНИИПО МЧС России. Москва, 2020. С. 380-387.

14. Катаева Л.Ю., Ильичева М.Н., Лощилов А.А. Математическое моделирование тушения лесного пожара капсулами с водой в термоактивной оболочке//Вычислительная механика сплошных сред. 2020. Т. 13. № 3. С. 320-336. DOI: 10.7242/1999-6691/2020.13.3.26 


\section{Reference}

1. Kopylov, N. P., Karpov, V. N., Kuznecov, A. E., Fedotkin, D. V., Hasanov, I. R., Sushkina, E. Ju., "Osobennosti tushenija lesnyh pozharov s primeneniem aviacii," Vestnik Tomskogo gosudarstvennogo universiteta, No. 59, 2019, pp. 79-86.

2. Glushkov, D. O., Kopylov, N. P., Kropotova, S. S., Kuznecov, G. V., Strizhak, P. A., Lokalizacija i podavlenie gorenija lesnyh materialov s primeneniem aviacii, Novosibirsk: Izd-vo SO RAN, 2020, 337 p.

3. Satoh, K., Maeda, I., Kuwahara, K., Yang, K., "A Numerical Study of Water Dump in Aerial Fire Fighting," Fire Safety Science, Vol. 8, 2005, pp. 777-787. doi:10.3801/IAFSS.FSS.8-777

4. Loshhilov, S. A., Maslenikov, D. A., Postnov, A. D., Kataeva, L. Ju., "Issledovanie vlijanija intensivnosti sbrosa vody na dinamiku lesnogo pozhara," Estestvennye i tehnicheskie nauki. No. 6 (68), 2013, pp. $37-40$.

5. Nishhenkov, V. V., Romanova, N. A., Kataeva, L. Ju., Maslennikov, D. A., Loshhilov, A. A., “Ob optimal'noj strategii tushenija lesnogo pozhara vodjanoj pushkoj," Sovremennye problemy nauki i obrazovanija, No. 3, 2014, p. 692.

6. Kataeva, L. Y., Maslennikov, D. A., Loshchilova, N. A. On the laws of combustion wave suppression by free water in a homogeneous porous layer of organic combustible materials," Fluid Dynamics, Vol. 51, No. 3, 2016, pp. 389-399.

7. Nakorjakov, V. E., Kuznecov, G. V., Strizhak, P. A., "O predel'nyh poperechnyh razmerah kapel'nogo oblaka pri razrushenii vodjanogo massiva v processe padenija s bol'shoj vysoty," Doklady akademii nauk, Vol. 475, No. 2, 2017, pp. 145-149. DOI: 10.7868/S0869565217020062

8. Flemmer, R. L. C. and Banks, C. L., "On the Drag Coefficient of a Sphere," Powder Technol., Vol. 48, 1986, pp. 217-221.

9. Turton, R. and Levenspiel, O. A., "A Short Note on the Drag Correlation for Spheres," Powder Technol., Vol. 47, No. 1, 1986, pp. 83-86.

10. Kataeva, L. Ju., Karpuhin, V. B., "O metode Gira chislennogo modelirovanija dinamicheskih sistem, opisyvaemyh zhestkimi obyknovennymi differencial'nymi uravnenijami," Nauka i tehnika transporta, No. 2. -M.: RGOTUPS, 2008.

11. Vojtkov, I. S., Volkov, R. S., Kuznecov, G. V., Strizhak, P. A., "Vysokotemperaturnoe isparenie kapel' vody v gazovoj srede,” Zhurnal tehnicheskoj fiziki, Vol. 87, No. 12, 2017, pp. 1911-191410.

12. Babkin, A. V., Chislennye metody v zadachah fiziki bystroprotekajushhih processov: uchebnik dlja vtuzov, M.: Izd-vo MGTU im. N. Je. Baumana, 2006. - 520 p.

13. Il'icheva, M. N., Kataeva, L. Ju., "Matematicheskoe modelirovanie tushenija lesnogo pozhara kapsulami s vodoj v razrushajushhejsja obolochke," XXXII nauchno-prakticheskaja konferencija. Aktual'nye problemy pozharnoj bezopasnosti, VNIIPO MChS Rossii. Moskva, 2020, pp. 380-387.

14. Kataeva, L. Ju., Il'icheva, M. N., Loshhilov, A. A., "Matematicheskoe modelirovanie tushenija lesnogo pozhara kapsulami s vodoj v termoaktivnoj obolochke," Vychislitel'naja mehanika sploshnyh sred, Vol. 13, No. 3, 2020, pp. 320-336. DOI: 10.7242/1999-6691/2020.13.3.26 